Review began 12/24/2021 Review ended 01/18/2022 Published 01/18/2022

\section{(๑) Copyright 2022}

Saha et al. This is an open access article distributed under the terms of the Creative Commons Attribution License CC-BY 4.0., which permits unrestricted use, distribution, and reproduction in any medium, provided the original author and source are credited.

\title{
Epicardial Fat Thickness: A Cardiometabolic Risk Marker in Rheumatoid Arthritis
}

\author{
Sekhar Saha ${ }^{1}$, Rajnish Singh ${ }^{1}$, Irfan A. Mir ${ }^{2}$, Naman Bansal ${ }^{2}$, Pankaj K. Singh ${ }^{3}$, Mir Nadeem ${ }^{4}$ \\ 1. Internal Medicine, Postgraduate Institute of Medical Education \& Research, Dr. Ram Manohar Lohia Hospital, New \\ Delhi, IND 2. Internal Medicine, School of Medical Sciences \& Research, Greater Noida, IND 3. Emergency Medicine, \\ School of Medical Sciences \& Research, Greater Noida, IND 4. Internal Medicine, King Khalid University and \\ Associated Hospital, Abha, SAU
}

Corresponding author: Irfan A. Mir, drirfan765742@gmail.com

\begin{abstract}
Background: Rheumatoid arthritis (RA) is a chronic, systemic, inflammatory disorder of unknown etiology which mainly involves synovial joints. However, the corresponding systemic inflammation may result in disorders of multiple other organ systems. Several organs and organ systems are potentially involved in RA, particularly in severe diseases. The organs most involved are the lung, heart, eyes, and nervous system. Extra-articular manifestations of RA may develop even before the onset of arthritis. Emerging epidemiological evidence shows that cardiovascular disease (CVD) accounts for near about 50\% of RAassociated death. Epicardial fat thickness (EFT) has recently emerged as a new marker of cardiometabolic risk. Although Epicardial fat (EF) is needed for heart muscle function, given its intrinsic inflammatory status, EFT displays the potential to serve as a therapeutic target in patients with RA.
\end{abstract}

Objectives: To evaluate EFT using echocardiography in RA patients compared to age and sex-matched control and to find the factors associated with EFT in RA patients.

Materials and methods: This current study was conducted in the Department of Medicine, Postgraduate Institute of Medical Education \& Research. The study was conducted from November 2016 to March 2018. Patients with $\mathrm{BMI} \geqslant 30 \mathrm{~kg} / \mathrm{m}^{2}$, diabetes mellitus, primary hyperlipidemia, and uncontrolled hypertension were particularly excluded. Thirty patients of age and sex-matched controls were also taken for the study. All the patients and controls selected for the study were subjected to detailed history taking and clinical examination. They were subjected to lab investigations and echocardiography. The 30 RA patients included in the study were diagnosed according to the 2010 ACR-EULAR criteria. Disease activity was measured by the disease activity score (DAS28) index.

Results: Group 1 included 30 patients with RA and group 2 included 30 age and sex-matched controls. Pearson correlation analysis was done between EFT and other variables. Only HDL, erythrocyte sedimentation rate (ESR), high-sensitivity C-reactive protein (hS-CRP), rheumatoid factor (RF), anti-cyclic citrullinated peptide (anti-CCP), DAS28, and disease duration were found to have a significant correlation with EFT.

Conclusion: In patients with RA, EFT, left ventricular mass, and diastolic dysfunction are increased in RA patients compared to healthy controls. Out of the conventional CVD risk factors, only HDL was associated with increased EFT in RA patients. In RA patients, DAS28, disease duration, RF, anti-CCP, and markers of inflammation (ESR, hs-CRP) were also associated with increased EFT.

Categories: Cardiology, Internal Medicine, Rheumatology

Keywords: disease duration, disease activity score, high density lipoprotein, cardiovascular risk factor, epicardial fat thickness, rheumatoid arthritis

\section{Introduction}

Rheumatoid arthritis (RA) is a chronic, systemic, inflammatory disorder of unknown etiology which mainly involves synovial joints. The estimated prevalence of RA in the community in India is $0.75 \%$ [1]. In RA, the subsequent inflammatory changes lead to cartilage and bone destruction. In addition to this, the corresponding systemic inflammation may result in disorders of multiple other organ systems [2]. Several organs and organ systems are potentially involved in RA, particularly in severe diseases. Extra-articular manifestations occur in about $40 \%$ of patients with RA over a lifetime of disease [3]. Other than joints, the organs most involved are the lung, heart, eyes, and nervous system. Extra-articular manifestations of RA may develop even before the onset of arthritis. Emerging epidemiological evidence shows that cardiovascular disease (CVD) accounts for near about 50\% of RA-associated death. Epicardial fat thickness (EFT) has recently emerged as a new marker of cardiometabolic risk [4]. Although epicardial fat (EF) is needed for heart muscle function, given its intrinsic inflammatory status, EFT displays the potential to serve as a therapeutic target in patients with RA [5]. 
Although studies on EFT in RA are very few, it is being suggested that EFT is increased in patients with RA and is associated with increased cardiovascular risk. Indian studies on EFT in RA are not available, and to our knowledge, it is the first study on EFT in RA patients. In our study, we aim to evaluate EFT using echocardiography (ECHO) in RA patients compared to age and sex-matched controls and to determine factors that are associated with EFT.

\section{Materials And Methods}

This current study was a hospital-based cross-sectional observational study, which was conducted in the Department of Medicine, Postgraduate Institute of Medical Education \& Research, Dr. Ram Manohar Lohia Hospital, New Delhi. The study was conducted from November 2016 to March 2018. The total number of patients included in the study was 30 patients. Patients aged $>18$ years were selected from the general outpatient department (OPD) and rheumatology clinic run by the department of medicine. Patients included in the study satisfied the inclusion and exclusion criteria and gave their written informed consent. Patients with body mass index (BMI) $\geqslant 30 \mathrm{~kg} / \mathrm{m}^{2}$, diabetes mellitus, primary hyperlipidemia, and uncontrolled hypertension were particularly excluded. Thirty patients of age and sex-matched controls were also taken for the study. All the issues including the ethical issues were evaluated by the Institutional Review Board and approved via notification number TP(MD/MS) (61/2016) /IEC/PGIMER/RMLH 7765/16 (Dated: 5/10/2016). All the patients and controls selected for the study were subjected to detailed history taking and clinical examination. They were subjected to lab investigations and ECHO. The 30 RA patients included in the study were diagnosed according to the 2010 American College of Rheumatology-European League Against Rheumatism (ACR-EULAR) criteria [6]. Disease activity was measured by the disease activity score (DAS28) index [7].

ECHO with pulse wave Doppler was performed in Echo Lab at the Department of Cardiology by a single cardiologist who was blind to patient details. A transthoracic two-dimensional (2D) ECHO examination was performed by PHILIPS HD 11XE machine, using the standard technique with study population in the left lateral decubitus position using a 3.5-MHz transducer. All the measurements were taken and interpreted following the American Society of Echocardiography recommendations [8]. The EFT was measured according to the method first described and validated by Iacobellis et al. [9]. The EF was identified as the echo-free space between the outer wall of the myocardium and the visceral layer of the pericardium. EFT was measured in the parasternal long-axis view, perpendicularly on the free wall of the right ventricle at endsystole in three cardiac cycles. Maximum EFT was measured at the point on the free wall of the right ventricle along the midline of the ultrasound beam, perpendicular to the aortic annulus, used as an anatomical landmark for this view. The average value of three cardiac cycles was considered [10].

Left ventricular mass (LVM) was similarly determined using an anatomically validated formula of Devereux et al. [11]:

LVM=0.8\{1.04[([LVEDD + IVSD + PWD $] 3$ - LVEDD3) $]\}+0.6$, where LVEDD, IVSD, and PWD represent left ventricular end-diastolic diameter, interventricular septal diameter, and posterior wall diameter in diastole, respectively, was derived assuming LV dimensions in centimeters. LVEDD, PWD, and IVSD were measured in parasternal long-axis view by M mode.

Left ventricular diastolic dysfunction was diagnosed and graded based on measurements of early (E wave) and late (A wave) diastolic mitral flow velocities, determined on pulsed wave Doppler. Continuous variables were presented as mean \pm SD. Categorical variables were presented as the number with percentage (\%) in the bracket. Statistical tests were applied as follows:

1. Quantitative variables were compared using an unpaired t-test between the two groups.

2. Qualitative variables were compared using the Chi-square test.

3. Pearson correlation was used to determine the association between two variables.

The data were entered in MS EXCEL spreadsheet and analysis was done using Statistical Package for Social Sciences (SPSS, Chicago, IL, USA) version 18.0. P-value <0.05 was considered statistically significant.

\section{Results}

The study population was divided into two groups for further description and analysis: group 1 included 30 patients with RA and group 2 included 30 age and sex-matched controls.

In Table 1, the two groups were comparable for age, sex, BMI, SBP, DBP, FBS, eGFR, TC, HDL, LDL, and TG. Erythrocyte sedimentation rate (ESR) and FBS were higher in cases compared to controls as expected. 


\section{Cureus}

\begin{tabular}{|c|c|c|c|}
\hline & Group $1(\mathrm{~N}=30)$ & Group $2(\mathrm{~N}=30)$ & P-value \\
\hline Age (years) & $40.97 \pm 7.44$ & $41.07 \pm 6.91$ & 0.96 \\
\hline $\operatorname{Sex}(m / f)^{\star}$ & $4(13 \%) / 26(87 \%)$ & $1(3 \%) / 29(97 \%)$ & 0.161 \\
\hline Body Mass Index (BMI) $\left(\mathrm{kg} / \mathrm{m}^{2}\right)$ & $23.93 \pm 2.73$ & $23.46 \pm 2.32$ & 0.47 \\
\hline Systolic Blood Pressure (SBP) (mm of Hg) & $120.60 \pm 10$ & $120 \pm 9.51$ & 0.81 \\
\hline Diastolic Blood Pressure (DBP) (mm of Hg) & $79.80 \pm 7.07$ & $79.27 \pm 6.53$ & 0.76 \\
\hline Fasting Blood Sugar (FBS) (mg/dL) & $85.06 \pm 7.29$ & $81.97 \pm 8.10$ & 0.13 \\
\hline Estimated Glomerular Filtration Rate (eGFR) (mL/min/1.73 $\left.\mathrm{m}^{2}\right)$ & $86.51 \pm 19.10$ & $89.65 \pm 25.18$ & 0.59 \\
\hline Total Cholesterol (TC) (mg/dL) & $176.80 \pm 41.76$ & $168.70 \pm 19.20$ & 0.34 \\
\hline Low-Density Lipoprotein (LDL) (mg/dL) & $100.17 \pm 25$ & $106.47 \pm 24.94$ & 0.33 \\
\hline High-Density Lipoprotein (HDL) (mg/dL) & $38 \pm 9.24$ & $38.77 \pm 7.50$ & 0.73 \\
\hline Triglycerides (TG) (mg/dL) & $122.17 \pm 36.79$ & $135.47 \pm 38.70$ & 0.18 \\
\hline Erythrocyte Sedimentation Rate (ESR) (mm) & $36.40 \pm 22.19$ & $15.90 \pm 6.85$ & $<0.001$ \\
\hline Disease Duration (years) & $4.75 \pm 2.21$ & - & \\
\hline Disease Activity Score (DAS28) & $2.89 \pm 0.84$ & - & \\
\hline Rheumatoid Factor（RF) (U/mL) & $16.90 \pm 7.44$ & - & \\
\hline Anti-Cyclic Citrullinated Peptide (Anti-CCP) (U/mL) & $104.22 \pm 92.89$ & - & \\
\hline high sensitive C Reactive Protein (hS-CRP) (mg/L) & $7.33 \pm 2.18$ & - & \\
\hline
\end{tabular}

\section{TABLE 1: Comparison of clinical characteristics in two groups}

Data have been expressed as Mean \pm SD unless stated otherwise. *Data expressed as a number with percentage inside bracket.

In Table 2, EFT, LVEDD, IVSD, LVM, and LVDD were higher in cases compared to controls. EF, which represents a systolic function, and posterior wall thickness at end-diastole were similar in the two groups.

\begin{tabular}{|c|c|c|c|}
\hline & Group $1(\mathrm{~N}=30)$ & Group $2(\mathrm{~N}=30)$ & P-value \\
\hline Epicardial Fat Thickness (EFT) (mm) & $5.08 \pm 0.79$ & $4.33 \pm 0.73$ & $<0.001$ \\
\hline Left Ventricular End Diastolic Dysfunction (LVEDD) (cm) & $4.97 \pm 0.67$ & $4.27 \pm 0.45$ & $<0.001$ \\
\hline Posterior Wall Thickness at end Diastole (PWD) ( $\mathrm{cm}$ ) & $0.87 \pm 0.06$ & $0.86 \pm 0.05$ & 0.22 \\
\hline Interventricular Septal Diameter (IVSD) (cm) & $0.89 \pm 0.07$ & $0.85 \pm 0.04$ & 0.01 \\
\hline Left Ventricular Mass (LVM) (gm) & $153.07 \pm 44.00$ & $112.20 \pm 23.13$ & $<0.001$ \\
\hline Ejection Fraction (EF) (\%) & $60.67 \pm 2.17$ & $61.67 \pm 2.40$ & 0.10 \\
\hline \multirow[t]{2}{*}{ Left Ventricular Diastolic Dysfunction (LVDD) } & Grade1-8 (26\%) & Grade1-4 (13\%) & 0.02 \\
\hline & Grade2-2 (7\%) & Grade2-0 (0\%) & 0.014 \\
\hline
\end{tabular}

TABLE 2: Comparison of echocardiographic parameters in two groups

Data have been expressed as Mean \pm SD unless stated otherwise. 


\section{Cureus}

Table 3 represents Pearson correlation analysis between EFT and other variables. Only high-density lipoprotein, ESR, high-sensitivity C-reactive protein (hS-CRP), rheumatoid factor (RF), anti-cyclic citrullinated peptide (anti-CCP), DAS28, and disease duration were found to have a significant correlation with EFT. However, no correlation was found between EFT and other conventional risk factors of CVD like age, sex, BMI, SBP, DBP, FBS, LDL, and triglycerides.

\begin{tabular}{|c|c|c|}
\hline & $\mathbf{R}$ & P-value \\
\hline Age (years) & 0.353 & 0.056 \\
\hline $\operatorname{Sex}(\mathrm{m} / \mathrm{f})$ & 0.087 & 0.647 \\
\hline Body Mass Index (BMI) (kg/m²) & 0.343 & 0.064 \\
\hline Systolic Blood Pressure (SBP) (mm of Hg) & 0.059 & 0.758 \\
\hline Diastolic Blood Pressure (DBP) (mm of Hg) & 0.32 & 0.084 \\
\hline Fasting Blood Sugar (FBS) (mg/dL) & 0.026 & 0.892 \\
\hline Triglycerides (TG) (mg/dL) & 0.288 & 0.123 \\
\hline High-Density Lipoprotein (HDL) (mg/dL) & -0.687 & $<0.001$ \\
\hline Low-Density Lipoprotein (LDL) (mg/dL) & 0.12 & 0.529 \\
\hline Dlsease Duration (years) & 0.443 & 0.014 \\
\hline Erythrocyte Sedimentation Rate (ESR) (mm) & 0.511 & $<0.001$ \\
\hline Rheumatoid Factor (RF) (U/mL) & 0.470 & 0.009 \\
\hline Anti-cyclic Citrullinated Peptide (Anti-CCP) (U/mL) & 0.413 & 0.023 \\
\hline high sensitivity C Reactive Protein (hS CRP) (mg/L) & 0.392 & 0.032 \\
\hline Disease Activity Score (DAS28) & 0.551 & 0.002 \\
\hline
\end{tabular}

TABLE 3: Correlation of epicardial fat thickness (EFT) with different factors

Figures 1, 2 showed that LVM and LVDD correlate positively with EFT, respectively. 


\section{Cureus}

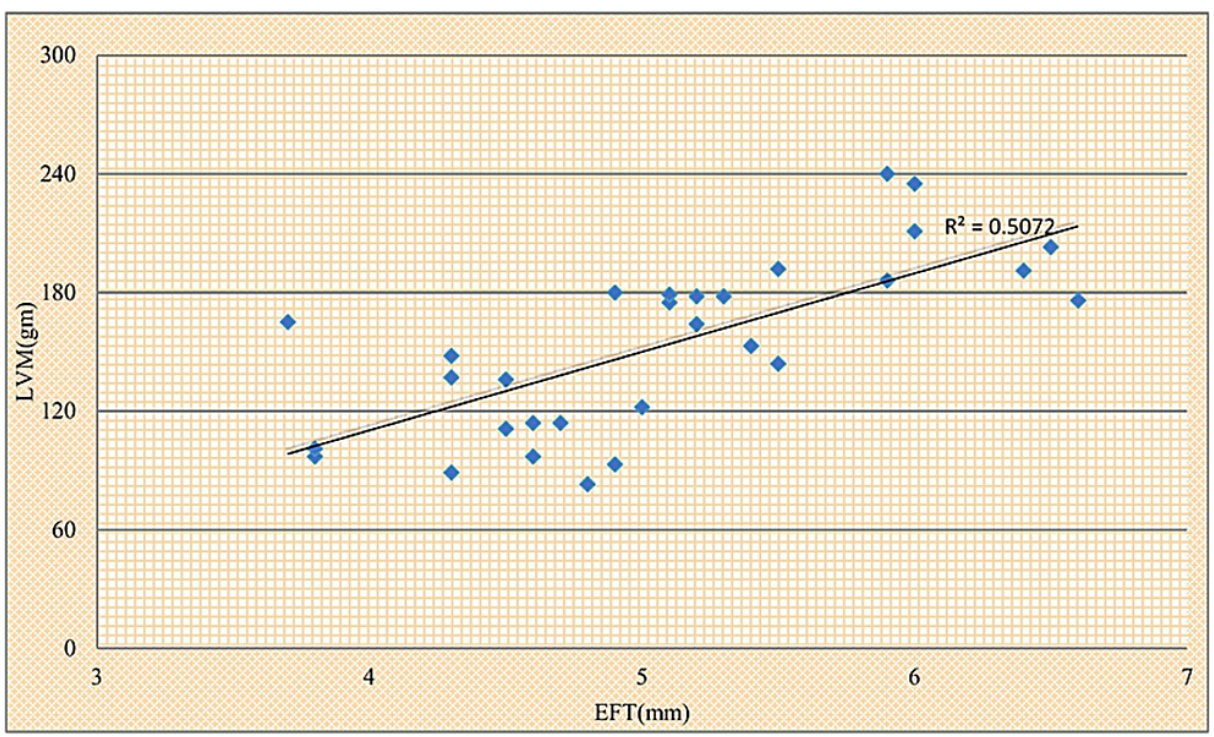

FIGURE 1: Scattered plot showing correlation of epicardial fat thickness (EFT) with left ventricular mass (LVM).

$r=0.712, p$-value $=<0.001$

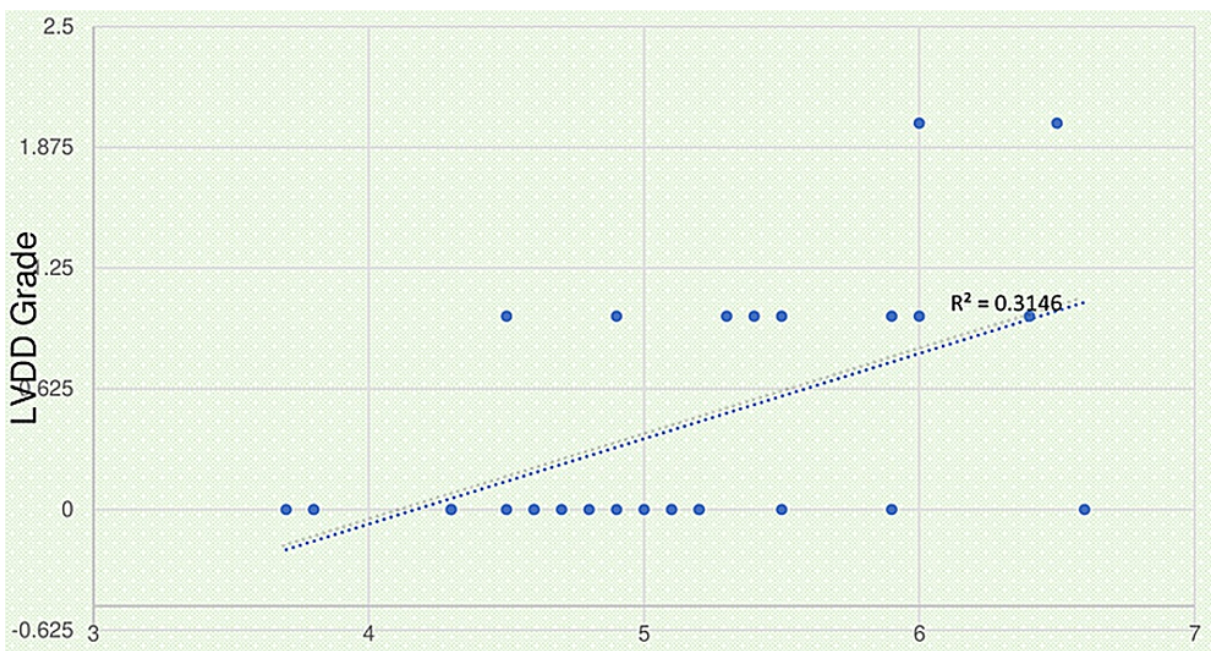

FIGURE 2: Scattered plot showing correlation of epicardial fat thickness (EFT) with left ventricular diastolic dysfunction (LVDD).

$r=0.561, p$-value $=0.001$

\section{Discussion}

Epicardial adipose tissue (EAT) is a proxy measure for visceral adiposity and visceral fat has been shown to be a reliable predictor of metabolic risk [12]. An increasing body of research suggests that EAT thickness is linked to traditional anthropometric and clinical factors such as BMI, waist circumference (WC), systolic blood pressure, and diastolic blood pressure [13,14]. The goal of this study was to compare EFT in RA patients to age and sex-matched controls, as well as to determine the variables linked to increased EFT in RA patients. The traditional risk factors have failed to explain the higher CVD mortality and morbidity in RA patients, and EFT is becoming more well recognized as a CVD risk.

We tested the study population for EFT, LVM, and LVDD using an ECHO with a color Doppler and also looked for traditional risk variables as well as disease status indicators including RF, anti-CCP, disease duration, 
DAS28, and inflammatory markers like ESR, hS-CRP. The EFT, LVDD, and LVM were also measured in the controls along with traditional cardiovascular risk variables. In the research group, the average EFT was 5.08 $\pm 0.79 \mathrm{~mm}$, with ranges ranging from $3.8 \mathrm{~mm}$ to $6.5 \mathrm{~mm}$. The average EFT in the control group was 4.33 $\pm 0.73 \mathrm{~mm}$, with ranges of $3.0 \mathrm{~mm}$ to $5.6 \mathrm{~mm}$. When compared to the control group, EFT was found to be considerably greater (p-value 0.001 ) in the study group (Table 1). Similar findings were obtained in studies by Iacobellis et al. [10], Natale et al. [15], Nelson et al. [16], and Lima-Martnez et al. [17]. Our study is the first of its kind in the Asian subcontinent with no studies in India so far.

In our study, we went a step further and used ECHO and Doppler to evaluate LVM in both groups, found that the average LVM in the study group was $153.07 \pm 44.00 \mathrm{~g}$ and in the control group was $112.20 \pm 23.13 \mathrm{~g}$, which was statistically significant (Table 2). Hence, we discovered that patients with RA had higher LVM than age and sex-matched controls and that when dispersed diagrams with EFT were drawn, EFT rose considerably and proportionally with LVM (Figure 1). This is consistent with the findings of Rudominer et al. [18] and Avina-Zubieta et al. [19].

In addition, $26 \%$ of patients in the study group developed grade one LVDD, compared to $13 \%$ in the control group (p-value 0.02 ). In addition, $7 \%$ of patients in the study group had grade two LVDD, whereas no one in the control group had grade two LVDD (p-value- 0.014). According to research conducted by Liang et al. [20], the prevalence of LVDD in patients with RA is high. They discovered that RA patients had a greater incidence of LVDD than healthy controls and that RA duration is also linked to LVDD. The LVDD has been seen in people with RA who do not have clinically significant heart dysfunction in previous research.

The age, sex, BMI, fasting blood sugar, lipid profile, blood pressure, and estimated glomerular filtration rate were compared and statistical analysis was performed between the two groups. Among them, FBS and ESR were determined to have significant differences between these two groups. Only HDL was linked to greater EFT in RA patients of the traditional CVD risk variables. Higher EFT was also linked to increased DAS28, disease duration, RF, anti-CCP, and inflammatory markers (ESR, hs-CRP) in RA patients (Table 3).

In the study group, Pearson correlation had been done between EFT as a dependant variable with conventional risk factors and markers of disease activity and markers of inflammations as independent variables in cases. It was discovered that EFT has a strong relationship with HDL (r-0.687, p-0.001), ESR (r0.511, p-0.001), hS-CRP (r-0.392, p-0.032), RF (r-0.470, p-0.009), anti-CCP (r-0.413, p-0.023), disease duration (r-0.443, p-0.014), DAS 28 (r-0.551, p-0.002) Table 3, LVM (r-0.712, p-<0.001) (Figure 1), and LVDD (r-0.561, p-0.001) (Figure 2).

\section{Limitations}

The primary limitation of our study was the small sample size. A small sample size has low statistical power and thus may yield false-negative results. The other limitation of our study it was a cross-sectional design, and the results cannot be generalized to the general population. Neither can we apply our results to the general population due to the numerous exclusion criteria?

Despite this, we believe that our findings provide a valuable contribution to the EFT and RA. Hence, future bigger and prospective studies are required to confirm our results.

\section{Conclusions}

In patients with RA, EFT, LVM, and diastolic dysfunction are increased in RA patients compared to healthy controls. The increased EFT is correlated positively with independent CVD risk factors like increased LVM and LVDD. Out of the conventional CVD risk factors, only HDL was associated with increased EFT in RA patients.

In RA patients, DAS28, disease duration, RF, anti-CCP, and markers of inflammation (ESR, hs-CRP) were also associated with increased EFT.

\section{Additional Information \\ Disclosures}

Human subjects: Consent was obtained or waived by all participants in this study. Institutional Review Board of Postgraduate Institute of Medical Education \& Research, Dr Ram Manohar Lohia Hospital, New Delhi issued approval TP(MD/MS) (61/2016)/IEC/PGIMER/RMLH 7765/16. Animal subjects: All authors have confirmed that this study did not involve animal subjects or tissue. Conflicts of interest: In compliance with the ICMJE uniform disclosure form, all authors declare the following: Payment/services info: All authors have declared that no financial support was received from any organization for the submitted work. Financial relationships: All authors have declared that they have no financial relationships at present or within the previous three years with any organizations that might have an interest in the submitted work. Other relationships: All authors have declared that there are no other relationships or activities that could appear to have influenced the submitted work. 


\section{References}

1. Rheumatoid arthritis. (2006). Accessed: August 19, 2016: http://www.mdguidelines.com/arthritisrheumat Medical Disability Guidelines.

2. McInnes IB, Schett G: The pathogenesis of rheumatoid arthritis . N Engl J Med. 2011, 365:2205-19. 10.1056/NEJMra1004965

3. Turesson C, O'Fallon WM, Crowson CS, Gabriel SE, Matteson EL: Extra-articular disease manifestations in rheumatoid arthritis: incidence trends and risk factors over 46 years. Ann Rheum Dis. 2003, 62:722-7. 10.1136/ard.62.8.722

4. Lima-Martínez MM, Blandenier C, Iacobellis G: Epicardial adipose tissue: more than a simple fat deposit? . Endocrinologia y Nutricion. 2013, 60:320-8.

5. Ormseth MJ, Lipson A, Alexopoulos N, et al.: Association of epicardial adipose tissue with cardiometabolic risk and metabolic syndrome in patients with rheumatoid arthritis. Arthritis Care Res (Hoboken). 2013, 65:1410-5. 10.1002/acr.22027

6. Aletaha D, Neogi T, Silman AJ, et al.: 2010 Rheumatoid arthritis classification criteria: an American College of Rheumatology/European League Against Rheumatism collaborative initiative. Arthritis Rheum. 2010, 62:2569-81. 10.1002/art.27584

7. Fries JF, Spitz P, Kraines RG, Holman HR: Measurement of patient outcome in arthritis . Arthritis Rheum. 1980, 23:137-45. 10.1002/art.1780230202

8. Lang RM, Badano LP, Mor-Avi V, et al.: Recommendations for cardiac chamber quantification by echocardiography in adults: an update from the American Society of Echocardiography and the European Association of Cardiovascular Imaging. . J Am Soc Echocardiogr 2015;28:1-39.e14.. https://asecho.org/wpcontent/uploads/2018/08/WFTF-Chamber-Quantification-Summary-Doc-Final-July-18.pdf,

9. Singh N, Singh H, Khanijoun HK, Iacobellis G: Echocardiographic assessment of epicardial adipose tissue - a marker of visceral adiposity. Mcgill J Med. 2007, 10:26-30.

10. Iacobellis G, Willens HJ: Echocardiographic epicardial fat: a review of research and clinical applications . J Am Soc Echocardiogr. 2009, 22:1311-9; quiz 1417-8. 10.1016/j.echo.2009.10.013

11. Devereux RB, Alonso DR, Lutas EM, Gottlieb GJ, Campo E, Sachs I, Reichek N: Echocardiographic assessment of left ventricular hypertrophy: comparison to necropsy findings. Am J Cardiol. 1986, 57:450-8. 10.1016/0002-9149(86)90771-X

12. Nguyen-Duy TB, Nichaman MZ, Church TS, Blair SN, Ross R: Visceral fat and liver fat are independent predictors of metabolic risk factors in men. Am J Physiol Endocrinol Metab. 2003, 284:E1065-71. 10.1152/ajpendo.00442.2002

13. Iacobellis G, Ribaudo MC, Assael F, et al.: Echocardiographic epicardial adipose tissue is related to anthropometric and clinical parameters of metabolic syndrome: a new indicator of cardiovascular risk. J Clin Endocrinol Metab. 2003, 88:5163-8. 10.1210/jc.2003-030698

14. Torres C, Lima-Mart inez MM, Rosa FJ, et al.: Epicardial adipose tissue and its association to plasma adrenomedullin levels in patients with metabolic syndrome. Endocrinolog 1a y Nutricio n. 2011, 58:401-8.

15. Natale F, Tedesco MA, Mocerino R, et al.: Visceral adiposity and arterial stiffness: echocardiographic epicardial fat thickness reflects, better than waist circumference, carotid arterial stiffness in a large population of hypertensives. Eur J Echocardiogr. 2009, 10:549-55. 10.1093/ejechocard/jep002

16. Nelson MR, Mookadam F, Thota V, et al.: Epicardial fat: an additional measurement for subclinical atherosclerosis and cardiovascular risk stratification?. J Am Soc Echocardiogr. 2011, 24:339-45. 10.1016/j.echo.2010.11.008

17. Lima-Martínez MM, Campo E, Salazar J, et al.: Epicardial fat thickness as cardiovascular risk factor and therapeutic target in patients with rheumatoid arthritis treated with biological and nonbiological therapies. Arthritis. 2014, 2014:782850. 10.1155/2014/782850

18. Rudominer RL, Roman MJ, Devereux RB, et al.: Independent association of rheumatoid arthritis with increased left ventricular mass but not with reduced ejection fraction. Arthritis Rheum. 2009, 60:22-9. 10.1002/art.24148

19. Aviña-Zubieta JA, Choi HK, Sadatsafavi M, Etminan M, Esdaile JM, Lacaille D: Risk of cardiovascular mortality in patients with rheumatoid arthritis: a meta-analysis of observational studies. Arthritis Rheum. 2008, 59:1690-7. 10.1002/art.24092

20. Liang KP, Myasoedova E, Crowson CS, et al.: Increased prevalence of diastolic dysfunction in rheumatoid arthritis. Ann Rheum Dis. 2010, 69:1665-70. 10.1136/ard.2009.124362 